\title{
Pictogram: A Better Tool for Counselling Tuberculosis Patients on Medication Use, Diet, and Life Style Modifications
}

\author{
By Veintramuthu Sankar* \\ Rinku Mary Joseph ${ }^{\dagger}$ \\ Pinky Mariyam Thomas \\ Paul C. Auguin ${ }^{+}$ \\ Ritto Thomas
}

\begin{abstract}
Relapse and spread of tuberculosis occurs due to lack of knowledge among the patients. India has $37 \%$ of the global total of illiterate patients. This emphasizes the need for developing effective tools to counsel patients according to their literacy level. So, we focus on developing pictograms which can be used to counsel even those TB patients with low literacy levels. To evaluate the role of pictograms in conveying information about medication use, diet, and life style modifications for TB patients and to develop a pictographic leaflet for counseling them. Study included patients with basic education diagnosed with respiratory diseases. In Phase I survey, the best out of 5 pictograms, which conveyed good understanding for each counselling point, were selected and modified. In Phase II, comprehensibility of these pictograms was tested using "guess-ability" and "translucency" studies. Best 56 pictograms out of 240 were selected by 20 pharmacists and 30 patients. 150 patients were enrolled in the guess-ability and translucency studies. Comprehensibility of pictograms did not vary based on gender, age group, or educational level. But differences existed for specific pictograms. Pictograms are generally well understood when the intended meanings were explained. Out of 56 pictograms, 26 passed the European Commission criteria. Pictograms play a vital role in educating patients irrespective of literacy. Pictographic leaflets can be used as an effective counseling aid in TB patients which help to improve compliance.
\end{abstract}

Keywords: Diet, Life style modifications, Medication use, Patient counseling, Pictogram, Tuberculosis

\section{Introduction}

Tuberculosis (TB) remains a major global health problem. The World Health Organization (WHO) has estimated that about 8.6 million people worldwide are infected with tuberculosis and each year 1.3 million people die of TB (WHO 2013, Raviglione and Uplikar 2006). Out of the global total of TB cases, 2.3 million were estimated to have occurred in India, which is approximately $30 \%$ of worlds TB burden (Government of India TB India 2014). Recent survey by WHO estimated that out of 9 million TB cases in the

\footnotetext{
* Vice Principal and Professor, Department of Pharmaceutics, PSG College of Pharmacy, TamilNadu, India.

${ }^{\dagger}$ Pharm D Intern, Department of Pharmacy Practice, PSG College of Pharmacy, TamilNadu, India.

${ }^{\ddagger}$ Pharm D Intern, Department of Pharmacy Practice, PSG College of Pharmacy, TamilNadu, India.

${ }^{+}$Pharm D Intern, Department of Pharmacy Practice, PSG College of Pharmacy, TamilNadu, India.

${ }^{\circ}$ Pharm D Intern, Department of Pharmacy Practice, PSG College of Pharmacy, TamilNadu, India.
} 
world 3 million patients have been left out without being treated. Most poignant fact to note here is that out of these 3 million cases, 1 million cases are from India (WHO 2013). But at the same time, India is the second largest Directly observed treatment, short course (DOTS) provider in the world (Bagchi et al. 2010).

In developing countries, patient adherence to the standard anti-tuberculosis therapy has been estimated to be as low as $40 \%$ and this disturbing fact thus becomes one of the major causes of treatment failure and emergence of drug resistant TB (Government of India TB India 2014). In addition, low patient adherence can increase the risk of treatment failure, relapses, deaths, and prolonged infection and thereby, it remains as a huge hurdle to the success of tuberculosis programmes (Vijay et al. 2010). Studies in different parts of the world revealed misconceptions and limited knowledge about the disease and its treatment (Maswanganyi et al. 2014). The probable reason for this could be lack of patient provider interaction and low literacy rate among the defaulting patients (Mohamed et al. 2007). This emphasizes the need for improving interpersonal communication skills of health providers, which enable them to impart knowledge about tuberculosis, and also designing and developing effective tools to counsel patients in tune with their literacy level.

Studies have also shown that non-adherence is more prevalent among illiterate patients (Vijay et al. 2010, Gopi et al. 2007). According to the EFA (Education for All) Global Monitoring Report 2013-14 commissioned by UNESCO, India has $37 \%$ of the global total of illiterate patients. This indicates the need for developing effective tools to counsel patients according to their literacy level. Approaches involving only written information directed to individual patient have been criticized for using language that is difficult to understand and interpret (Bachrach et al. 1998). The readability of adult and pediatric patient education materials are often too advanced for the general adult population (Dowse and Ehlers 2005).

As pictures convey information more effectively than words, pictograms can be a better tool for educating and counseling patients with lower literacy rate. It can reduce the barriers associated with poor health literacy, as they have been shown to be superior to text in attracting attention, stimulating motivation, and enhancing recall. These pictograms are expected to be understood by nearly everybody as they can communicate equally and effectively to speakers of a wide variety of languages (Dowse and Ehlers 2005, Korenevsky et al. 2013, Revol et al. 2013).

In pharmaceutical settings, they have been shown to increase comprehension, information recall, and adherence to treatment and can be used to help bridge communication barriers due to varying levels of health literacy, language differences, and cultural variation (Tijus et al. 2007, Houts et al. 2001). Very few studies have been done about pictograms that help in counseling tuberculosis patients.

Since poor knowledge, attitudes and practices about $\mathrm{TB}$ have been reported by several international studies (Sockrider and Wolle 2005), we focused on designing and developing pictograms which can be used to counsel 
TB patients on medication use, diet and life style modifications, and to identify whether comprehension of pictogram related to TB counseling depends on factors such as gender, age group, and level of education.

\section{Methodology and Design}

This prospective - observational study was conducted at a 900 bedded multispecialty teaching hospital located in the southern Indian state of Tamil Nadu. The study was done in two consecutive phases in order to identify and modify the pictograms that are better understood by the patients.

A total of 180 patients and 20 pharmacists were recruited for the study. The inclusion criteria was in-patients aged 18 yrs and above with only basic education (below $8^{\text {th }} \mathrm{std}$ ), who were diagnosed with any respiratory disease. Participants were excluded from participating if they were blind or had impaired ability to visualize images. Other categories of patients excluded were psychiatric patients, pregnant women, and severely ill patients.

\section{Phase I: Survey}

Phase I (survey) was carried out using 20 pharmacists and 30 patients to select the best 2 , out of 5 pictograms showing better understanding for each counseling point. The initially used pictograms ( 5 pictures for each counseling point) were designed by the investigators. These pictograms depicted counseling on medication usage (time of administration and adverse drug reactions), diet and lifestyle modifications in tuberculosis. The selection of 2 out of 5 was based on the preference given by maximum number of the participants. Modifications were made to the pictograms based on the suggestions from pharmacists and difficulties faced by the patients in understanding them. The difficulties were: lack of clarity, misinterpretation of pictogram, lack of colored pictograms, and the recommendation made by the patients to include real sketch diagrams instead of stick-man images. The selected pictograms, after relevant modifications, were used to carry out the phase II study. The selected pictograms were divided into 3 categories: life style modifications (20 pictograms - 10 counseling points), drug use (20 pictograms - 10 counseling points), and diet (16 pictograms - 8 counseling points).

\section{Phase II : One-On-One Interview}

Phase II was done to further evaluate and validate the most guessed pictograms from phase I. This was done using 2 validated methods: transparency (guess-ability) and translucency. Transparency is "the guessability of the meaning" of a picture when its significance is unknown to the participants (Houts and Doak 2006). Translucency is the degree to which the participant believes that the image represents what it is suppose to portray, 
after its meaning is being explained to him/her (Houts and Doak 2006, Yovetich and Young 1988).

In guess-ability study, the pictograms selected were shown to the patients and the response to the pictograms were recorded using a 3 point visual analog Likert scale as incorrect, partially correct, and correct (Revol et al. 2013). To assess the translucency, participants were told the intended meaning of the pictogram and asked to rate how well the image represented its meaning on a 5-point visual analog Likert scale as no relation, slight relation, moderate relation, strong relation, and exact relation (Revol et al. 2013). A score of 1 indicated that there was no relationship between the pictogram and its meaning, whereas a score of 5 indicated an exact relationship.

The protocol of the study was approved by the Institutional Human Ethics Committee of our hospital. Informed consent was obtained from all the participants prior to participating. Patients diagnosed with respiratory diseases were interviewed personally and data regarding social habits, level of education, and occupation were collected.

For the purpose of the analysis, the participants were stratified based on gender, age group, and level of education: Gender - Male/Female; Age group 18-64 yrs/65 yrs \& above; and Level of education - Illiterate/Primary (up to $5^{\text {th }}$ std)/Middle (up to $8^{\text {th }}$ std).

The different variables on which the patients were categorised were statistically analysed using SPSS Version 19 (Revol et al. 2013). To fulfil the criteria from the guidelines issued by European Commission, the pictograms must be correctly guessed by $80 \%$ or more participants for guess-ability test and $80 \%$ of participants must grade the pictograms with a score of 3 or more on the Likert scale for translucency test (Dickinson et al. 2001, Mansoor and Dowse 2003). For each sub analysis based on gender, age group or literacy level, only pictograms with less than $80 \%$ correct answer were addressed in the result section.

\section{Results}

\section{Phase I}

The survey was carried out to select two sets of pictograms with greater percentage of appeal and understanding. The selected set of pictograms was used for Phase II study. A total of 20 pharmacists and 30 patients were recruited randomly. The patients varied in age groups and levels of education. Details of participants in phase I are shown in Table 1. 
Table 1. Details of the Participants in Phase I Survey

\begin{tabular}{|c|c|c|c|}
\hline \multicolumn{4}{|l|}{ Patient participants } \\
\hline Demographic data & Category & No. of participants & Percentage (\%) \\
\hline \multirow[t]{2}{*}{ Gender } & Male & 17 & 56.6 \\
\hline & Female & 13 & 43.3 \\
\hline \multirow[t]{2}{*}{ Age group } & $18-64$ yrs & 18 & 60 \\
\hline & 65 yrs \& above & 12 & 40 \\
\hline \multirow[t]{3}{*}{ Level of education } & Illiterates & 9 & 30 \\
\hline & Primary (up to $5^{\text {th }}$ std) & 13 & 43.3 \\
\hline & Middle (up to $8^{\text {th }}$ std) & 8 & 26.6 \\
\hline \multicolumn{4}{|c|}{ Pharmacist participants } \\
\hline Demographic data & Category & No. of participants & Percentage (\%) \\
\hline \multirow[t]{2}{*}{ Gender } & Male & 9 & 45 \\
\hline & Female & 11 & 55 \\
\hline
\end{tabular}

Out of five pictograms, 2 pictograms were selected for each counseling point (20 pictograms for medication use, 20 for life style modifications, and 16 for diet). These selected pictograms were preferred by maximum number of participants and were used to carry out phase II study. The other pictograms with lower acceptance were rejected. Modifications suggested by the pharmacists and patients are included in Table 2.

Table 2. Modifications Suggested During Phase I Survey

Change the following pictograms into coloured ones:

- Medication use: Rashes, Red urine.

- Life style modifications: No smoking, Avoid Crowd, Eye check up.

- Diet: Wheat, Carrot, Orange, Apple, Fruits, Vegetables.

Use real sketch diagrams instead of stick man images for the following:

- Medication use: Itching, Stomach ache, Headache.

- Life style modifications: Good sleep, Eye check-up, Avoid crowd.

$\checkmark$ Include the picture of cow in the pictogram depicting milk.

$\checkmark$ Include real cigarette images instead of black and white images.

$\checkmark \quad$ Instead of using the image of vomiting into closet, use simple vomiting images.

$\checkmark$ Avoid nausea pictograms as it is confusing with stomach ache pictograms.

$\checkmark$ Use pictograms depicting man drinking alcohol instead of pictograms which depicted only alcohol bottles.

$\checkmark$ Include rooster images in the pictogram which depicted morning.

$\checkmark$ Use specific pictograms for each fruits and vegetables instead of depicting a group of fruits and vegetables.

The necessary changes were made to the pictograms based on above suggestions before carrying out the phase II study. Pictograms selected from phase I to phase II study are given in Figure 1. 
Vol. 4, No. 1 Sankar et al.: Pictogram: A Better Tool for Counselling Tuberculosis...

Figure 1. Pictograms Selected from Phase I Survey for Phase II Study Medication use

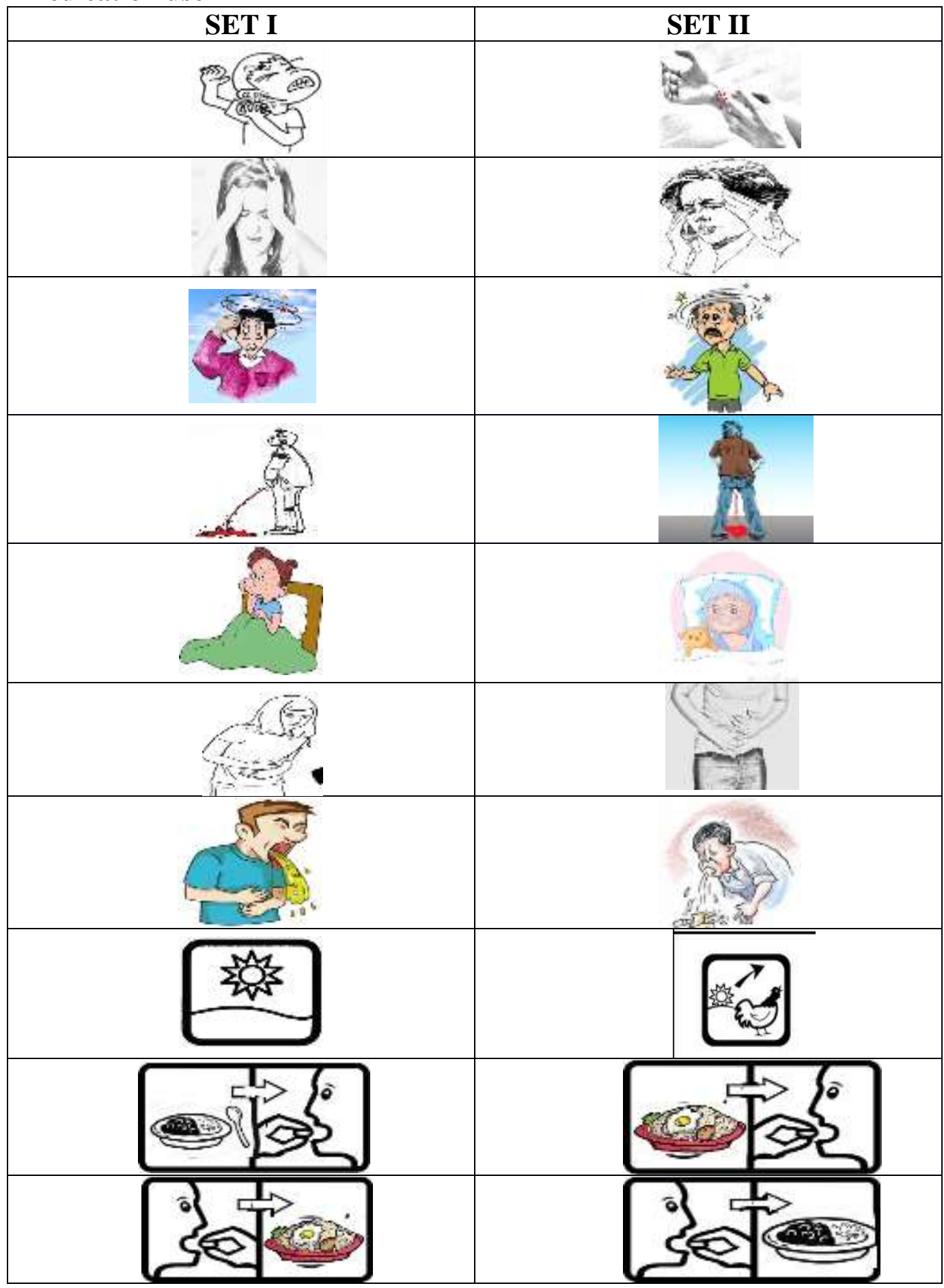


Life style modifications

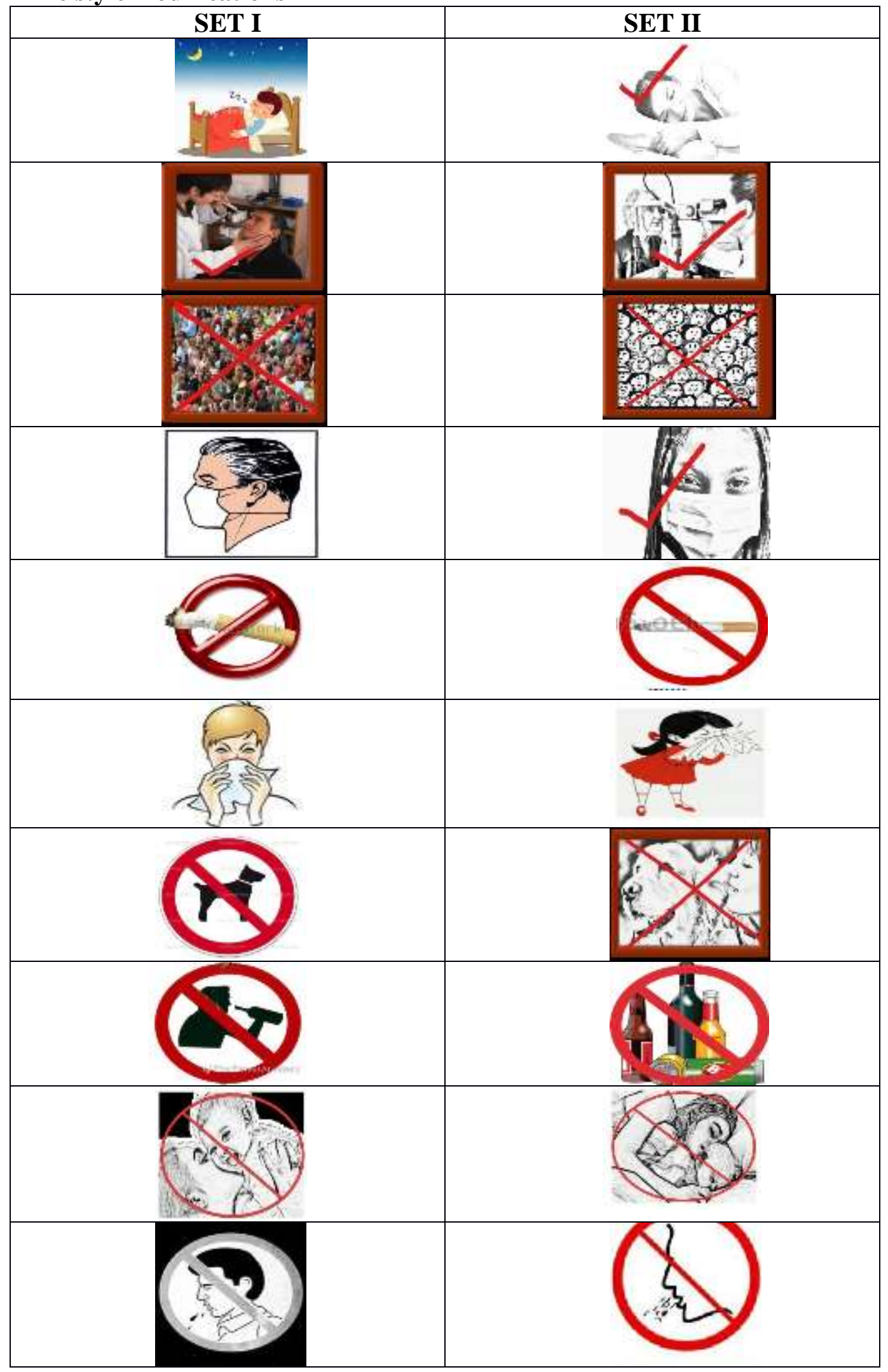




\section{Diet}

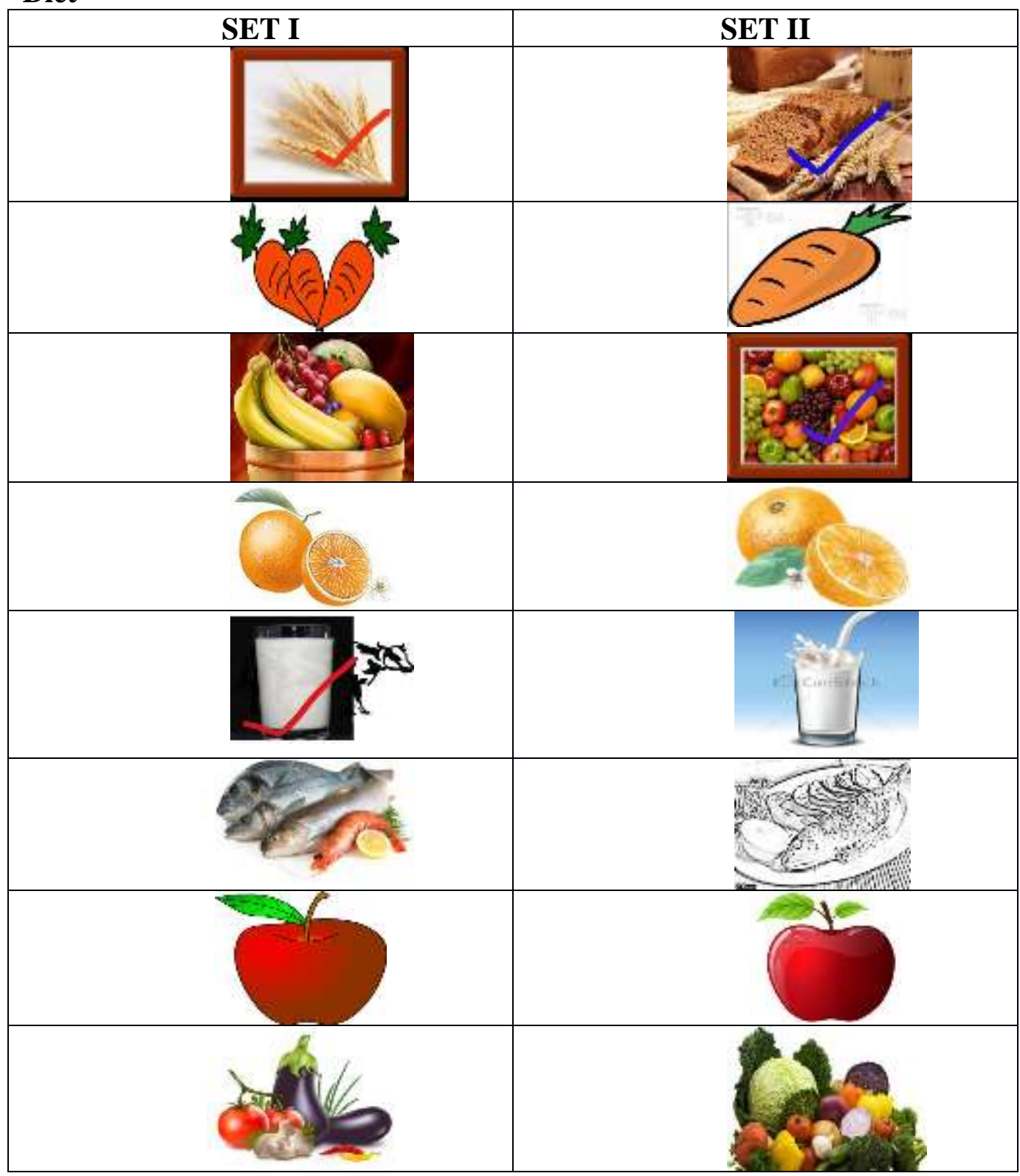

\section{Phase II}

A total of 150 patients with basic level of education (below $8^{\text {th }}$ std) and diagnosed with any respiratory disease were recruited for the guess-ability and translucency study. Out of these 150 patients, the pictograms related to drug use, life style modifications, and diet were shown to 50 patients each. Demographics of patients enrolled for guess-ability and translucency are listed in Table 3. 
Table 3. Details of the Patients Enrolled for Guess-ability and Translucency

\begin{tabular}{|c|c|c|c|}
\hline \multicolumn{4}{|c|}{ Pictograms for Medication use } \\
\hline Demographic data & Category & No. of patients $(n=50)$ & Percentage (\%) \\
\hline \multirow[t]{2}{*}{ Gender } & Male & 28 & 56 \\
\hline & Female & 22 & 44 \\
\hline \multirow[t]{2}{*}{ Age group } & $18-64$ yrs & 33 & 66 \\
\hline & 65 yrs \& above & 17 & 34 \\
\hline \multirow[t]{3}{*}{ Level of education } & Illiterate & 14 & 28 \\
\hline & Primary (up to $5^{\text {th }}$ std) & 13 & 26 \\
\hline & Middle (up to $8^{\text {th }}$ std) & 23 & 46 \\
\hline \multicolumn{4}{|c|}{ Pictograms for Life style modifications } \\
\hline Demographic data & Category & No. of patients $(n=50)$ & Percentage (\%) \\
\hline \multirow[t]{2}{*}{ Gender } & Male & 35 & 70 \\
\hline & Female & 15 & 30 \\
\hline \multirow[t]{2}{*}{ Age group } & $18-64$ yrs & 21 & 42 \\
\hline & 65 yrs \& above & 29 & 58 \\
\hline \multirow[t]{3}{*}{ Level of education } & Illiterate & 24 & 48 \\
\hline & Primary (up to $5^{\text {th }}$ std) & 22 & 44 \\
\hline & Middle (up to $8^{\text {th }}$ std) & 4 & 8 \\
\hline \multicolumn{4}{|l|}{ Pictograms for Diet } \\
\hline Demographic data & Category & No. of patients $(n=50)$ & Percentage (\%) \\
\hline \multirow[t]{2}{*}{ Gender } & Male & 35 & 70 \\
\hline & Female & 15 & 30 \\
\hline \multirow[t]{2}{*}{ Age group } & $18-64$ yrs & 39 & 78 \\
\hline & 65 yrs \& above & 11 & 22 \\
\hline \multirow[t]{3}{*}{ Level of education } & Illiterate & 23 & 46 \\
\hline & Primary (up to $5^{\text {th }}$ std) & 8 & 16 \\
\hline & Middle (up to $8^{\text {th }}$ std) & 19 & 38 \\
\hline
\end{tabular}

\section{Transparency Test (Guess-ability)}

The overall guess-ability was higher for diet related pictograms $(82.3 \%)$ compared to medication use $(73.7 \%)$ and life style modifications $(59.6 \%)$ related pictograms.

For medication use related pictograms, the average percentage of correct answers for set 2 pictograms $(80.6 \%)$ was higher than set 1 pictograms $(66.8 \%)$. Only $8 \%$ of the patients guessed correctly the pictogram which showed itching and in set 2 , the least percentage $(58 \%)$ was for the pictogram depicting the instruction "before food".

In case of life style modification related pictograms, the average percentage of correct answer for set 1 pictograms (61.6\%) was higher than that of set 2 pictograms $(57.6 \%)$. In set 1 , least percentage (42\%) of correct answer was for avoid pets and in set 2 only $4 \%$ patients guessed eye check up correctly.

For diet related pictograms, set 1 of pictograms (83\%) had higher guessability rate than set 2 pictograms $(81.7 \%)$. In both sets, only $40 \%$ patients guessed the pictogram depicting milk correctly. Pictograms guessed correctly by greater than $80 \%$ of participants are shown in Table 4 . 
Table 4. Pictograms Guessed Correctly by Greater than $80 \%$ of Participants

\section{$\checkmark$ Medication use:}

- Set 1: Head ache (80\%), Red colour urine (96\%), Stomach ache (94\%), Vomiting (100\%), Morning (92\%), Before food (82\%).

- Set 2: Head ache $(88 \%)$, Red colour urine $(100 \%)$, Stomach ache (86\%), Vomiting (90\%), Morning (98\%), After food (82\%).

$\checkmark$ Life style modifications:

- $\quad$ Set 1: Good sleep (100\%), Mask 1 (84\%), No smoking (82\%).

- Set 2: Do not spit (100\%), Mother and child (82\%).

$\checkmark$ Diet:

- Set 1: Fruits (96\%), Orange (82\%), Apple (96\%), Vegetable (100\%), Fish $(100 \%)$.

- Set 2: Fruits (98\%), Orange (84\%), Apple (100\%), Vegetable (96\%), Fish $(96 \%)$.

\section{Comparison of Pictogram Guess-ability}

The overall guess-ability score percentage is shown in Table 5.

\section{Male vs Female}

There was no statistically significant difference in overall guess-ability (all pictogram included) between male and female. However for pictograms which were guessed correctly by less than $80 \%$ of patients, only the following pictograms showed significant difference (details not included in tables or figures).

Dizzy 2: (57.1\% vs 54.5\%); p-value (0.0254); Avoid sleeping with child 1: Male (51.4\% vs 40\%); p-value (0.0054); Milk 1: (48.5\% vs 20\%); p-value (0.0418); Carrot 2: (71.4\% vs 93.3\%); p-value (0.0425).

\section{8-64 yrs vs 65 yrs \& above}

Overall guess-ability did not show any statistically significant difference. But for the pictograms which were guessed correctly by less than $80 \%$ of participants, only the following showed significant difference (details not included in tables or figures).

Dizzy 2: (60.6\% vs 47\%); p-value (0.0194); Mother \& child 1: 18-64yrs (57\% vs 81\%); p-value (0.016); Milk 2: (46.1\% vs 18.1\%); p-value (0.0121).

\section{Illiterate $v s$ Primary $v s$ Middle Education}

Comparing the patients based on their level of education, the overall guessability did not show any statistically significant difference. But for the pictograms which were guessed correctly by less than $80 \%$ of participants, only the following showed significant difference (details not included in tables or figures).

Itch 1: (0\% vs 7\% vs 13\%); p-value (0.0098); Eyecheck up 2: (6.25\% vs $4.5 \%$ vs $25 \%)$, p-value (0.0456); Milk 1: (30.4\% vs $12.5 \%$ vs $63.1 \%)$, p-value (0.0047); Milk 2: (30.4\% vs $12.5 \%$ vs 63.1\%), p-value (0.0174); Apple 1: (100\% vs $75 \%$ vs $100 \%)$, p-value (0.0031). 
Table 5. Overall Guess-ability Score Percentage

\begin{tabular}{|c|c|c|c|c|c|}
\hline \multicolumn{6}{|c|}{ Medication use } \\
\hline $\begin{array}{l}\text { Demographic } \\
\text { data }\end{array}$ & Category & $\begin{array}{c}\text { No of } \\
\text { patients } \\
(n=50)\end{array}$ & $\begin{array}{c}\text { Average } \\
\text { score } \\
\text { (out of } 2 \text { ) }\end{array}$ & $\begin{array}{c}\text { Score } \\
\text { Percentage } \\
(\%)\end{array}$ & $p$-value \\
\hline Gender & $\begin{array}{l}\text { Male } \\
\text { Female }\end{array}$ & $\begin{array}{l}28 \\
22\end{array}$ & $\begin{array}{l}1.56 \\
1.52\end{array}$ & $\begin{array}{l}78 \\
76\end{array}$ & 0.8481 \\
\hline Age group & $\begin{array}{c}18-64 \text { yrs } \\
65 \text { yrs \& } \\
\text { above }\end{array}$ & $\begin{array}{l}33 \\
17\end{array}$ & $\begin{array}{l}1.54 \\
1.54\end{array}$ & $\begin{array}{l}77 \\
77\end{array}$ & 0.9991 \\
\hline $\begin{array}{l}\text { Level of } \\
\text { education }\end{array}$ & $\begin{array}{c}\text { Illiterate } \\
\text { Primary } \\
\text { (up to } 5^{\text {th }} \text { std) } \\
\text { Middle } \\
\text { (up to } 8^{\text {th }} \text { std) }\end{array}$ & $\begin{array}{l}14 \\
13 \\
23\end{array}$ & $\begin{array}{l}1.45 \\
1.57 \\
1.59\end{array}$ & $\begin{array}{l}72.5 \\
78.5 \\
79.5\end{array}$ & 0.8169 \\
\hline \multicolumn{6}{|c|}{ Life style modifications } \\
\hline $\begin{array}{l}\text { Demographic } \\
\text { data }\end{array}$ & Category & $\begin{array}{c}\text { No of } \\
\text { patients } \\
(n=50)\end{array}$ & $\begin{array}{c}\text { Average } \\
\text { score } \\
\text { (out of 2) }\end{array}$ & $\begin{array}{c}\text { Score } \\
\text { Percentage } \\
(\%)\end{array}$ & $p$-value \\
\hline Gender & $\begin{array}{c}\text { Male } \\
\text { Female }\end{array}$ & $\begin{array}{l}35 \\
15\end{array}$ & $\begin{array}{l}1.44 \\
1.37\end{array}$ & $\begin{array}{c}72 \\
68.5\end{array}$ & 0.7889 \\
\hline Age group & $\begin{array}{c}18-64 \text { yrs } \\
65 \text { yrs \& } \\
\text { above }\end{array}$ & $\begin{array}{l}21 \\
29\end{array}$ & $\begin{array}{l}1.34 \\
1.45\end{array}$ & $\begin{array}{c}67 \\
72.5\end{array}$ & 0.8233 \\
\hline $\begin{array}{l}\text { Level of } \\
\text { education }\end{array}$ & $\begin{array}{c}\text { Illiterate } \\
\text { Primary } \\
\text { (up to } 5^{\text {th }} \text { std) } \\
\text { Middle } \\
\text { (up to } 8^{\text {th }} \text { std) }\end{array}$ & $\begin{array}{l}24 \\
22 \\
4\end{array}$ & $\begin{array}{c}1.429 \\
1.42 \\
1.368\end{array}$ & $\begin{array}{l}71 \\
71 \\
68\end{array}$ & 0.7335 \\
\hline \multicolumn{6}{|l|}{ Diet } \\
\hline $\begin{array}{l}\text { Demographic } \\
\text { data }\end{array}$ & Category & $\begin{array}{c}\text { No of } \\
\text { patients } \\
(n=50)\end{array}$ & $\begin{array}{c}\text { Average } \\
\text { score } \\
\text { (out of } 2 \text { ) }\end{array}$ & $\begin{array}{c}\text { Score } \\
\text { Percentage } \\
(\%)\end{array}$ & $p$-value \\
\hline Gender & $\begin{array}{c}\text { Male } \\
\text { Female }\end{array}$ & $\begin{array}{l}35 \\
15\end{array}$ & $\begin{array}{l}1.74 \\
1.67\end{array}$ & $\begin{array}{l}87 \\
83\end{array}$ & 0.6718 \\
\hline Age group & $\begin{array}{c}18-64 \text { yrs } \\
65 \text { yrs \& } \\
\text { above }\end{array}$ & $\begin{array}{l}39 \\
11\end{array}$ & $\begin{array}{l}1.74 \\
1.63\end{array}$ & $\begin{array}{c}87 \\
81.5\end{array}$ & 0.5346 \\
\hline $\begin{array}{l}\text { Level of } \\
\text { education }\end{array}$ & $\begin{array}{c}\text { Illiterate } \\
\text { Primary } \\
\text { (up to } 5^{\text {th }} \text { std ) } \\
\text { Middle } \\
\text { (up to } 8^{\text {th }} \text { std) }\end{array}$ & $\begin{array}{c}23 \\
8 \\
\\
19\end{array}$ & $\begin{array}{l}1.65 \\
1.61 \\
1.83\end{array}$ & $\begin{array}{l}82.5 \\
80.5 \\
91.5\end{array}$ & 0.4587 \\
\hline
\end{tabular}


Translucency Test

Comparing the 3 category of pictograms, greater percentage of patients scored exact relation in the Likert scale for the pictograms depicting diet $(87.45 \%)$ medication use (80\%) and life style modifications $(71.6 \%)$.

The average score of strong relation and exact relation was higher in set 2 pictograms $(86.4 \%)$ compared to set 1 pictograms $(73.6 \%)$ in medication use. In set 1 , only $10 \%$ patients scored as exact relation in the Likert scale for the pictograms depicting itching and in set 2 , lowest score was for dizziness and before food $(38 \%)$.

In life style modifications, the average score of strong relation and exact relation was higher in set 1 pictograms $(73.8 \%)$ compared to set 2 pictograms (69.4\%). Only $42 \%$ patients scored exact relation for the pictogram showing avoid pets in set 1 and only $4 \%$ for eye check up in set 2 .

The average score of strong relation and exact relation was higher in set 2 pictograms $(88.2 \%)$ compared to set 1 pictograms $(86.7 \%)$ in diet. In both the sets, the pictogram depicting milk was scored exact relation by least number of patients (17\% and 40\%).

Pictograms scored as strong and exact relation by greater than $80 \%$ of participants are shown in Table 6.

Table 6. Pictograms Scored as Strong and Exact Relation by Greater than $80 \%$ of Participants

\section{$\checkmark$ Medication use:}

- Set 1: Headache $1(96 \%)$, Red colour urine (100\%), Stomach ache (96\%), Vomiting (100\%), Morning (92\%), Before food (86\%).

- Set 2: Itch (88\%), Head ache (96\%), Stomach ache (88\%), Red urine (100\%), Rash (94\%), Vomiting (94\%), Morning (94\%), After food (86\%).

\section{$\checkmark \quad$ Life style modifications:}

- Set 1: Good sleep (100\%), Eye check-up (80\%), Mask (84\%), No smoking (94\%).

- Set 2: Good sleep (92\%), Mother and child 2 (86\%), Do not spit (100\%).

\section{Diet:}

- Set 1: Wheat (80\%), Carrot (82\%), Fruits (96\%), Orange (90\%), Apple (98\%), Vegetable (100\%), Fish (100\%).

- Set 2: Carrot (82\%), Fruits (98\%), Orange (96\%), Apple (100\%), Vegetable $(98 \%)$, Fish (98\%). 


\section{Comparison of Pictogram Translucency}

The overall translucency score percentage is shown in Table 7.

Table 7. Overall Translucency Score Percentage

\begin{tabular}{|c|c|c|c|c|c|}
\hline \multicolumn{6}{|c|}{ Medication use } \\
\hline $\begin{array}{l}\text { Demographic } \\
\text { data }\end{array}$ & Category & $\begin{array}{c}\text { No of } \\
\text { patients } \\
(n=50)\end{array}$ & $\begin{array}{c}\text { Average } \\
\text { score }\end{array}$ & $\begin{array}{c}\text { Score } \\
\text { Percentage } \\
(\%)\end{array}$ & $p$-value \\
\hline Gender & $\begin{array}{c}\text { Male } \\
\text { Female }\end{array}$ & $\begin{array}{l}28 \\
22 \\
\end{array}$ & $\begin{array}{c}1.8 \\
1.77\end{array}$ & $\begin{array}{c}90 \\
88.5\end{array}$ & 0.8155 \\
\hline Age group & $\begin{array}{c}18-64 \text { yrs } \\
65 \text { yrs \& } \\
\text { above }\end{array}$ & $\begin{array}{l}33 \\
17\end{array}$ & $\begin{array}{l}1.78 \\
1.78\end{array}$ & $\begin{array}{l}89 \\
89\end{array}$ & 0.9569 \\
\hline $\begin{array}{l}\text { Level of } \\
\text { education }\end{array}$ & $\begin{array}{c}\text { Illiterate } \\
\text { Primary } \\
\text { (up to } 5^{\text {th }} \text { std) } \\
\text { Middle } \\
\text { (up to } 8^{\text {th }} \text { std) }\end{array}$ & $\begin{array}{l}14 \\
13 \\
23\end{array}$ & $\begin{array}{l}1.73 \\
1.79 \\
\\
1.81\end{array}$ & $\begin{array}{l}86.5 \\
89.5 \\
90.5\end{array}$ & 0.8401 \\
\hline \multicolumn{6}{|c|}{ Life style modifications } \\
\hline $\begin{array}{l}\text { Demographic } \\
\text { data }\end{array}$ & Category & $\begin{array}{c}\text { No of } \\
\text { patients } \\
(n=50)\end{array}$ & $\begin{array}{c}\text { Average } \\
\text { score }\end{array}$ & $\begin{array}{c}\text { Score } \\
\text { Percentage } \\
(\%)\end{array}$ & $p$-value \\
\hline Gender & $\begin{array}{c}\text { Male } \\
\text { Female }\end{array}$ & $\begin{array}{l}35 \\
15\end{array}$ & $\begin{array}{l}1.86 \\
1.86\end{array}$ & $\begin{array}{l}93 \\
93 \\
\end{array}$ & 0.546 \\
\hline Age group & $\begin{array}{c}18-64 \text { yrs } \\
65 \text { yrs \& } \\
\text { above }\end{array}$ & $\begin{array}{l}21 \\
29\end{array}$ & $\begin{array}{l}1.84 \\
1.87\end{array}$ & $\begin{array}{c}92 \\
93.5\end{array}$ & 0.558 \\
\hline $\begin{array}{l}\text { Level of } \\
\text { education }\end{array}$ & $\begin{array}{c}\text { Illiterate } \\
\text { Primary } \\
\text { (up to } 5^{\text {th }} \text { std) } \\
\text { Middle } \\
\text { (up to } 8^{\text {th }} \text { std) }\end{array}$ & $\begin{array}{l}24 \\
22 \\
4\end{array}$ & $\begin{array}{l}1.88 \\
1.85 \\
\\
1.81\end{array}$ & $\begin{array}{c}94 \\
92.5 \\
90.5\end{array}$ & 0.423 \\
\hline \multicolumn{6}{|l|}{ Diet } \\
\hline $\begin{array}{l}\text { Demographic } \\
\text { data }\end{array}$ & Category & $\begin{array}{c}\text { No of } \\
\text { patients } \\
(n=50)\end{array}$ & $\begin{array}{c}\text { Average } \\
\text { score }\end{array}$ & $\begin{array}{c}\text { Score } \\
\text { Percentage } \\
(\%)\end{array}$ & p-value \\
\hline Gender & $\begin{array}{c}\text { Male } \\
\text { Female }\end{array}$ & $\begin{array}{l}35 \\
15\end{array}$ & $\begin{array}{l}1.86 \\
1.82 \\
\end{array}$ & $\begin{array}{l}93 \\
91 \\
\end{array}$ & 0.7350 \\
\hline Age group & $\begin{array}{c}18-64 \text { yrs } \\
65 \text { yrs \& } \\
\text { above }\end{array}$ & $\begin{array}{l}39 \\
11\end{array}$ & $\begin{array}{l}1.87 \\
1.80\end{array}$ & $\begin{array}{c}93.5 \\
90\end{array}$ & 0.5473 \\
\hline $\begin{array}{l}\text { Level of } \\
\text { education }\end{array}$ & $\begin{array}{c}\text { Illiterate } \\
\text { Primary } \\
\text { (up to } 5^{\text {th }} \text { std) } \\
\text { Middle } \\
\text { (up to } 8^{\text {th }} \text { std) }\end{array}$ & $\begin{array}{c}23 \\
8 \\
19\end{array}$ & $\begin{array}{l}1.81 \\
1.85 \\
\\
1.92\end{array}$ & $\begin{array}{c}90 \\
92.5 \\
96\end{array}$ & 0.5396 \\
\hline
\end{tabular}


Vol. 4, No. $1 \quad$ Sankar et al.: Pictogram: A Better Tool for Counselling Tuberculosis...

Table 8. Guess-ability vs Translucency

Medication use

\begin{tabular}{|c|c|c|c|c|c|}
\hline Demographic data & Category & No of patients $(n=50)$ & Score percentage of guess-ability (\%) & $\begin{array}{c}\text { Score Percentage of } \\
\text { translucency (\%) }\end{array}$ & $p$-value $(* * *)$ \\
\hline Gender & $\begin{array}{c}\text { Male } \\
\text { Female }\end{array}$ & $\begin{array}{l}28 \\
22\end{array}$ & $\begin{array}{l}78 \\
76\end{array}$ & $\begin{array}{c}90 \\
88.5 \\
\end{array}$ & $\begin{array}{l}<0.0001 \\
<0.0001\end{array}$ \\
\hline Age group & $\begin{array}{c}18-64 \text { yrs } \\
65 \text { yrs \& above }\end{array}$ & $\begin{array}{l}33 \\
17 \\
\end{array}$ & $\begin{array}{l}77 \\
77 \\
\end{array}$ & $\begin{array}{l}89 \\
89 \\
\end{array}$ & $\begin{array}{l}<0.0001 \\
<0.0001 \\
\end{array}$ \\
\hline Level of education & $\begin{array}{c}\text { Illiterate } \\
\text { Primary (up to } 5^{\text {th }} \text { std) } \\
\text { Middle (up to } 8^{\text {th }} \text { std) }\end{array}$ & $\begin{array}{l}14 \\
13 \\
23 \\
\end{array}$ & $\begin{array}{l}72.5 \\
78.5 \\
79.5 \\
\end{array}$ & $\begin{array}{l}86.5 \\
89.5 \\
90.5 \\
\end{array}$ & $\begin{array}{l}<0.0001 \\
<0.0001 \\
<0.0001\end{array}$ \\
\hline \multicolumn{6}{|c|}{ Life style modifications } \\
\hline Demographic data & Category & No of patients $(n=50)$ & Score percentage of guess-ability (\%) & $\begin{array}{c}\text { Score Percentage of } \\
\text { translucency (\%) }\end{array}$ & $p$-value $(* * *)$ \\
\hline Gender & $\begin{array}{c}\text { Male } \\
\text { Female }\end{array}$ & $\begin{array}{l}35 \\
15 \\
\end{array}$ & $\begin{array}{c}72 \\
68.5 \\
\end{array}$ & $\begin{array}{l}93 \\
93 \\
\end{array}$ & $\begin{array}{l}<0.0001 \\
<0.0001 \\
\end{array}$ \\
\hline Age group & $\begin{array}{c}18-64 \text { yrs } \\
65 \text { yrs \& above }\end{array}$ & $\begin{array}{l}21 \\
29 \\
\end{array}$ & $\begin{array}{c}67 \\
72.5 \\
\end{array}$ & $\begin{array}{c}92 \\
93.5 \\
\end{array}$ & $\begin{array}{l}<0.0001 \\
<0.0001 \\
\end{array}$ \\
\hline Level of education & $\begin{array}{l}\text { Illiterate } \\
\text { Primary (up to } 5^{\text {th }} \text { std) } \\
\text { Middle (up to } 8^{\text {th }} \text { std) }\end{array}$ & $\begin{array}{c}24 \\
22 \\
4 \\
\end{array}$ & $\begin{array}{l}71 \\
71 \\
68 \\
\end{array}$ & $\begin{array}{c}94 \\
92.5 \\
90.5 \\
\end{array}$ & $\begin{array}{c}<0.0001 \\
<0.0001 \\
0.0047 \\
\end{array}$ \\
\hline \multicolumn{6}{|l|}{ Diet } \\
\hline Demographic data & Category & No of patients $(n=50)$ & Score percentage of guess-ability (\%) & $\begin{array}{c}\text { Score Percentage of } \\
\text { translucency }(\%)\end{array}$ & $p$-value (***) \\
\hline Gender & $\begin{array}{c}\text { Male } \\
\text { Female }\end{array}$ & $\begin{array}{l}35 \\
15 \\
\end{array}$ & $\begin{array}{c}87 \\
83.5 \\
\end{array}$ & $\begin{array}{l}93 \\
91 \\
\end{array}$ & $\begin{array}{l}<0.0001 \\
0.0005\end{array}$ \\
\hline Age group & $\begin{array}{c}18-64 \text { yrs } \\
65 \text { yrs \& above }\end{array}$ & $\begin{array}{l}39 \\
11 \\
\end{array}$ & $\begin{array}{c}87 \\
81.5 \\
\end{array}$ & $\begin{array}{c}93.5 \\
90 \\
\end{array}$ & $\begin{array}{l}<0.0001 \\
0.0030 \\
\end{array}$ \\
\hline Level of education & $\begin{array}{c}\text { Illiterate } \\
\text { Primary (up to } 5^{\text {th }} \text { std) } \\
\text { Middle (up to } 8^{\text {th }} \text { std) }\end{array}$ & $\begin{array}{c}23 \\
8 \\
19 \\
\end{array}$ & $\begin{array}{l}82.5 \\
80.5 \\
91.5 \\
\end{array}$ & $\begin{array}{c}90 \\
92.5 \\
96 \\
\end{array}$ & $\begin{array}{l}<0.0001 \\
0.0025 \\
0.0025 \\
\end{array}$ \\
\hline
\end{tabular}

Notes: $* * *$ Correlation is significant at the level of 0.05 (2 tailed). 
Male vs Female

There is no statistically significant difference in overall translucency (all pictogram included) between males and females in all the 3 categories of pictograms, that is, medication use, life style modifications and diet. Significant difference was not found even when each pictogram was compared between males and females (details not included in tables or figures).

\section{8-64 yrs vs 65 yrs \& above}

Comparing the overall translucency (all pictogram included) between the age group, no statistically significant difference was found in all the 3 categories of pictograms. None of the pictograms showed statistically significant difference between 18-64 yrs and 65 yrs \& above even when each pictogram was compared (details not included in tables or figures).

\section{Illiterate vs Primary vs Middle Education}

Comparing the patients based on their level of education, the overall translucency did not show any statistically significant difference. But for the pictograms which were scored as strong or exact relation by less than $80 \%$ of participants, only the following showed significant difference (details not included in tables or figures).

Dizzy 1: (28.5\% vs $100 \%$ vs $43.4 \%)$, p-value (0.0161); Avoid pet 2: (88\% vs $95.23 \%$ vs $100 \%)$, p-value (0.0395); Mask 1: (100\% vs $95.23 \%$ vs $100 \%)$, pvalue (0.005); No smoking 2: (100\% vs $95.23 \%$ vs $100 \%)$, p-value (0.01); Mother \& child 2: (92\% vs 66.66\% vs 100\%), p-value (0.001); Milk 1: (34.7\% vs $25 \%$ vs $73.6 \%$ ), p-value (0.0129); Milk 2: (39.1\% vs $62.5 \%$ vs $84.2 \%$ ), pvalue (0.0129).

\section{Guess-ability vs Translucency}

The overall score percentage of guess-ability $v s$ translucency is shown in Table 8 .

\section{Gender}

\section{Male patients}

Significance was found in all the 3 categories of pictograms comparing the overall scores of males in guess-ability and translucency: Medication use: $78 \%$ vs $90 \%$ (p-value <0.0001); Life style modifications: $72 \%$ vs $93 \%$ ( $\mathrm{p}$-value 0.0197); Diet: $87 \%$ vs $93 \%$ (p-value <0.0001).

\section{Female patients}

Significance was found in all the 3 categories of pictograms comparing the overall scores of females in guess-ability and translucency: Medication use: 76\% vs $88.5 \%$ (p-value <0.0001); Life style modifications: $68.5 \%$ vs $93 \%$ (pvalue 0.0114); Diet: $83.5 \%$ vs $91 \%$ (p-value 0.0005 ). 
Age Group

$\underline{18-64 \mathrm{yrs}}$

Significance was found in all the 3 categories of pictograms comparing the overall scores of 18-64 yrs in guess-ability and translucency. Medication use: 77\% vs $89 \%$ (p-value <0.0001); Life style modifications: $67 \%$ vs $93 \%$ (p-value 0.0101); Diet: $87 \%$ vs $93.5 \%$ (p-value 0.004).

65 yrs \& above

When the overall scores of 65 yrs \& above in guess-ability and translucency were compared, statistically significant differences were found. Medication use: $77 \%$ vs $89 \%$ (p-value <0.0001); Life style modifications: $72.5 \%$ vs $93 \%$ (p-value 0.0200); Diet: $81.5 \%$ vs 90 (p-value 0.0002).

\section{Level of Education}

Statistically significant differences were found when the overall guess-ability score was compared against the overall translucency score in patients with every level of education included in the study.

Illiterates

Medication use: $72.5 \%$ vs $86.5 \%$ (p-value <0.0001); Life style modifications: $71 \%$ vs $94 \%$ (p-value 0.0130); Diet: $82.5 \%$ vs $90 \%$ (p-value 0.0002).

Primary education

Medication use: $78.5 \%$ vs $88.5 \%$ (p-value 0.0003); Life style modifications: $71 \%$ vs $92.5 \%$ (p-value 0.0216); Diet: $80.5 \%$ vs $92.5 \%$ (p-value 0.0008).

Middle education

Medication use: $79.5 \%$ vs 78\% (p-value <0.0001); Life style modifications: $68 \%$ vs $90.5 \%$ (p-value 0.0176); Diet: $91.5 \%$ vs $96 \%$ (p-value $0.0001)$.

\section{Pictograms Selected from this Study for Preparing Leaflet}

From the guess-ability and translucency studies, the following pictograms were selected for preparing a leaflet as it was guessed correctly by $>80 \%$ of participants during the guess-ability study and scored as strong or exact relation on the Likert scale by $>80 \%$ of participants in the translucency study (European Commission Criteria).

Medication use: stomach ache 1, vomiting 1, before food 1, headache 2, red colour urine 2, morning 2, after food 2. Life style modifications: Good sleep 1, mask 1, no smoking1, mother and child 2, do not spit 2. Diet: vegetable 1, fish 1 , fruits 2 , orange 2 , apple 2 . 


\section{Discussion and Conclusion}

\section{Discussion}

Inadequate knowledge about tuberculosis emerged as a risk factor for a majority of therapy regimen of TB patients. Approaches involving only written information for individual patients use language that is difficult to understand and interpret (Bachrach et al. 1998). Our study helps in designing leaflets containing pictograms, which can act as an effective tool for counseling tuberculosis patients even with low literacy levels.

A total of 56 pictograms were selected from 240 pictograms through phase I survey. 26 pictograms were guessed correctly by $>80 \%$ population and 34 pictograms were scored 3 or above by $>80 \%$ of population. 16 pictograms failed both the tests. It may be necessary to redesign these failed ones using participant suggestions for improvement and further evaluation.

In order for pictograms to be understood by people, they have to relate to the population in which they will be used. The context of culture, and as we have discovered, plays a significant role in increasing patients' acceptance and understanding. Research has demonstrated that patients prefer locally developed pictograms (Dowse and Ehlers 2005). So we preferred developing pictograms by implementing suggestions of the patients themselves.

The results of the study showed that pictograms are generally well understood even in patients with low literacy rate. Moreover, the pictograms for diet counseling have higher guess-ability rates $(82.3 \%)$ as compared to life style modifications $(56.1 \%)$ and medication use $(73.7 \%)$. Pictograms are more readily interpreted when they convey a simple message (Houts et al. 1998, 2001). The study also supported the statement that simple pictograms depicting actions and themes such as vomiting, headache, apple, orange, "do not spit" were easy to understand as compared to complex anatomical pictograms such as eye checkup, itching, rashes etc. (Radhakrishnan et al. 2012).

But some of the common pictograms such as "do not smoke" and "do not drink alcohol" had lower guess-ability rates compared to other uncommon pictograms. This may be due to the guilty feeling of most smokers and alcoholics, which restricted them to accept the intended meaning of these pictograms and thus may have biased the results.

Although personal preferences for pictograms do not always differ, differences do exist for specific pictograms depending on an individual's education level, gender or age group, especially for pictograms which were guessed correctly by less than $80 \%$ participants (Itching, dizziness, eye checkup, milk). Similar results are seen in other studies which suggest that when communicating specific health and medication information with patients, pictograms may be interpreted differently or understood with different clarity depending on the individual (Richlera et al. 2012, Wilson et al. 2012).

Previous research has demonstrated that level of education can have a significant influence on the interpretation of certain pictograms, and that patients with higher level of education typically show improved pictogram comprehension 
(Dowse and Ehlers 2005). Therefore, our study included patients with only basic education, so that the developed pictograms can be understood equally by all patients without any discrimination in terms of education.

A strong relationship between literacy and pictogram comprehension was found mainly in pictograms which were guessed correctly by $<80 \%$ of study population, where the patients with middle education had guess-ability rates higher than illiterates. In guess-ability and Translucency, the younger population (18-64 yrs) was able to guess and could understand the pictograms better $(87 \%)$ as compared to those $65 \mathrm{yrs} \&$ above $(81.5 \%)$. This can be due to the difficulties faced by the elderly people like low level of education, occurrence of other co-morbidities etc.

The results also indicated that the pictograms are generally well understood by patients when the intended meanings were explained which is proved from the significant difference between guess-ability v/s translucency score. Similar results were found in other studies where the average recall assessment score was greater in the text with symbol group $(\mathrm{M}=6.65)$ as compared to symbol only group ( $M=6.36)$ (King et al. 2012).

This also supports the notion that pictograms are more easily interpreted by patients when they are accompanied with text or oral explanation. This validates FIP WHO statement on labeling which recommends inclusion of written explanation with pictograms for their use in medical settings (Knapp et al. 2005).

\section{Conclusion}

This study concludes that pictograms play a vital role in educating patients and can be used as an effective counseling aid, especially in low-literacy groups. Moreover, comprehension and recall of information can be improved by using verbal or written explanation to the pictograms while counseling the patients.

\section{Practice Implications}

We have developed a leaflet containing the pictograms from our guessability and translucency studies (Figure 2), which could be used as an effective counseling aid for TB patients with low literacy levels. These pictograms can communicate equally and effectively to speakers of a wide variety of languages and reduces the barriers associated with educational level. Use of pictographic leaflets to counsel TB patients and their family can improve compliance to anti-TB therapy, which in turn prevents the development of MDR TB and treatment failure. 
Figure 2. A Developed Leaflet

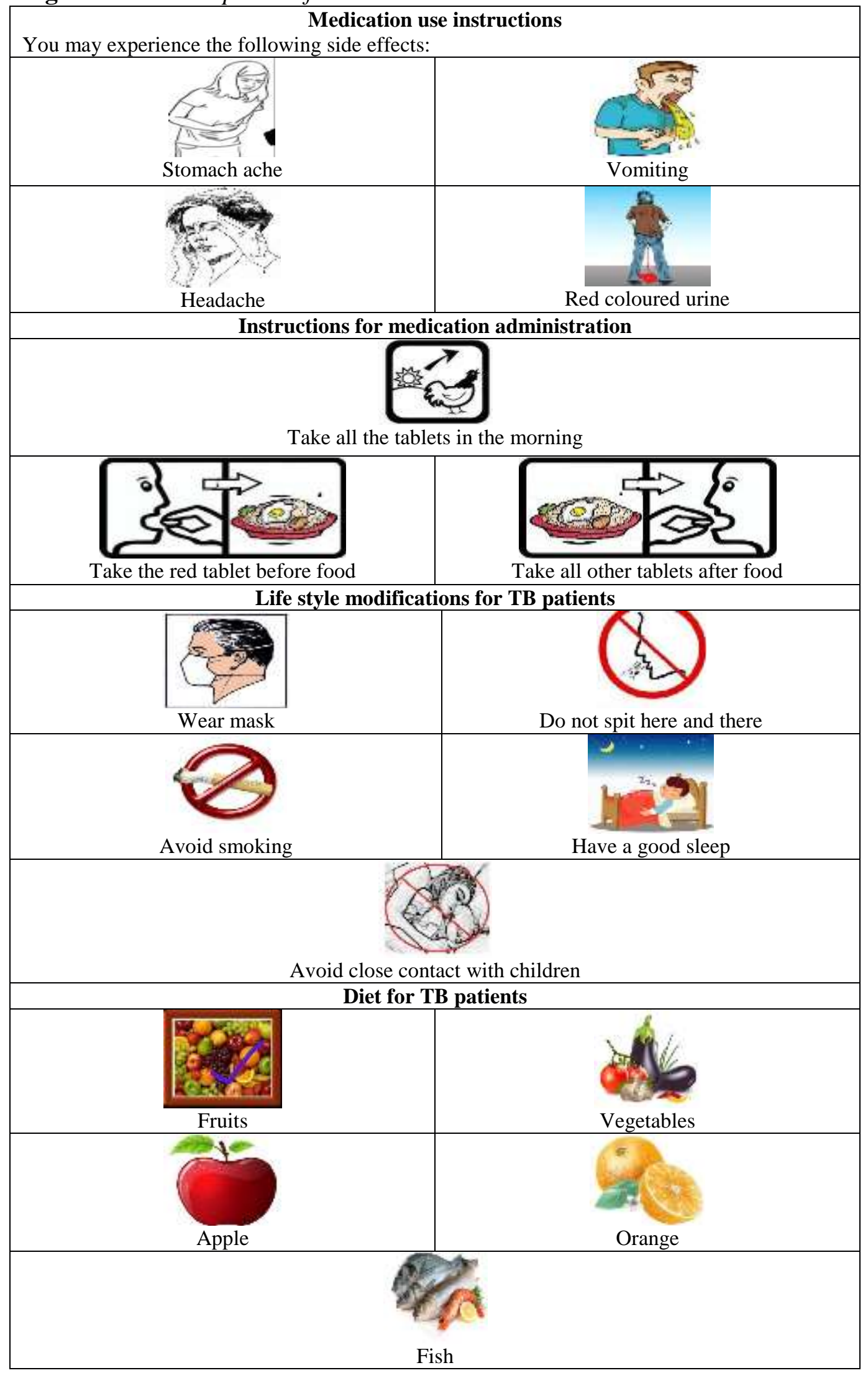




\section{Acknowledgement}

We would like to thank PSG College of Pharmacy, PSG hospitals, clinical sites, and the patients for their support and co-operation. We thank Dr. Sivaram Hariharan for the English proof reading and editing services.

\section{References}

Bachrach R, Houts PS, Witmer JT, Tringali CA, Bucher JA, Localio RA (1998) Using pictographs to enhance recall of spoken medical instructions. Patient Education Counseling 35: 83-88.

Bagchi S, Ambe G, Sathiakumar N (2010) Determinants of poor adherence to anti-TB therapy in Mumbai, India. International Journal of Preventive Medicine 1(4): 223-232.

Dickinson D, Raynor DK, Duman M (2001) Patient information leaflets for medicines: using consumer testing to determine the most effective design. Patient Education Counseling 43: 147-59.

Dowse R, Ehlers M (2005) Medicine labels incorporating pictograms: do they influence understanding and adherence? Patient Education Counseling 58(1): 63-70.

Gopi PG, Vasantha M, Muniyandi M, Chandrasekaran V, Balasubramanian R, Narayanan PR (2007) Risk factors for non-adherence to directly observed treatment in a rural TB unit, South India. Indian Journal of Tuberculosis 54: 66-70.

Government of India - TB India (2014) Revised national TB control program Annual Status Report 29.

Houts PS, Doak CC (2006) The role of pictures in improving health communication: a review of research on attention, comprehension, recall and adherence. Patient Education Counseling 61: 173-190.

Houts PS, Bachrach R, Witmer JT, Tringali CA, Bucher JA, Localio RA (1998) Using pictographs to enhance recall of spoken medical instructions. Patient Education Counseling 35: 83-88.

Houts PS, Witmer JT, Egeth HE, Loscalzo MJ, Zabora JR (2001) Using pictographs to enhance recall of spoken medical instructions II. Patient Education Counseling 43: 231-242.

King SR, McCaffrey III DJ, Bentley JP, Bouldin A, Hallam J, Wilkin NE (2012) The Influence of Symbols on the Short-Term Recall of PharmacyGenerated Prescription Medication Information in a Low Health Literate Sample. Journal of Health Communication 17: 280-293.

Knapp P, Raynor DK, Jebar AH, Price SJ (2005) Interpretation of medication pictograms by adults in the UK. Annals Pharmacotherapy 39(7-8): 12271233.

Korenevsky A, Vaillancourt R, Pouliot A, Revol M, Steed E, Besançon L (2013) How many words does a picture really tell? Cross sectional 
descriptive study of pictogram evaluation by youth. The Canadian Journal of Hospital Pharmacy 66(4): 219-26.

Mansoor LE, Dowse R (2003) Effect of pictograms on readability of patient information materials. Annals Pharmacotherapy 37: 1003-1009.

Maswanganyi NV, Lebese RT, Mashau NS, Khoza LB (2014) Patient perceived factor contributing to low TB cure rates at greater Giyani health care facilities. Health SA Gesondheid - Journal of Interdisciplinary Health Sciences 19(1). DOI: 10.4102/hsag.v19i1.724.

Mohamed AI, Yousif MA, Ottoa P, Bayoumi A (2007) Knowledge of TB: A survey among TB patients in Omdurman, Sudan. Sudanese Journal of Public Health 2(1): 217-225.

Revol M, Vaillancourt R, Pouliot A (2013) Evaluation, validation, and modification of pictograms depicting potential side effects to medication. Journal of Communication in Healthcare 6(2): 79-89.

Radhakrishnan R, Sudha V, Danturulu MV, Shreekant S (2012) Design and evaluation of pictograms for communicating information about adverse drug reactions to antiretroviral therapy in Indian human immunodeficiency virus positive patients. Journal of Pharmaceutical and Biomedical Sciences 16(10).

Raviglione M, Uplikar MW (2006) WHOs new stop TB strategy. The Lancet 367(9514): 952-955.

Richlera M, Vaillancourtf R, Celettib SJ, Besançonc L, Arund KP, Sebastiene F (2012) The use of pictograms to convey health information regarding side effects and/or indications of medications. Journal of Communication in Healthcare 5(4): 220-226.

Sockrider MM, Wolle JM (2005) Helping patients better adhere to treatment regimen. Journal of Respiratory Diseases 17: 204-216.

Tijus C, Barcenilla J, de Lavalette BC, Meunier JG (2007) The design, understanding and usage of pictograms. In. D Alamargot, P Tellier, J-M Cellier (Eds.), Written Documents in the Workplace p. 17-32. Brill Online.

Vijay S, Kumar P, Chauhan LS, Vollepore BH, Kizhakkethil UP, Rao SG (2010) Risk factors associated with default among new smear positive TB patients treated under DOTS in India. PLoS One 5(4): e10043. DOI: 10.1371 journal pone. 0010043 .

WHO (2013) Global Tuberculosis Report 2013. Chapter 1, 2.

Wilson EAH, Vaillancourt R, Pascuet E, Besançon LJR, Wolf MS (2012) Seeking international consensus in the use of icons for medication instructions. Journal of Communication in Healthcare 5(1): 67-72.

Yovetich W, Young TA (1988) The effects of representativeness and concreteness on the guessability of blissymbols. Augmentative and Alternative Communication 4: 35-39. 
